14 Halasi M, Gartel AL. A novel mode of FoxM1 regulation: positive autoregulatory loop. Cell Cycle 2009; 8: 1966-1967.

15 Attar EC, Johnson JL, Amrein PC, Lozanski G, Wadleigh M, DeAngelo DJ et al. Bortezomib added to daunorubicin and cytarabine during induction therapy and to intermediate-dose cytarabine for consolidation in patients with previously untreated acute myeloid leukemia age 60 to 75 years: CALGB (Alliance) study 10502. J Clin Oncol 2013; 31: 923-929.
16 Liu S, Liu Z, Xie Z, Pang J, Yu J, Lehmann E et al. Bortezomib induces DNA hypomethylation and silenced gene transcription by interfering with Sp1/NF-KBdependent DNA methyltransferase activity in acute myeloid leukemia. Blood 2008; 111: 2364-2373.

17 Gusarova GA, Wang IC, Major ML, Kalinichenko VV, Ackerson T, Petrovic V et al. A cell-penetrating ARF peptide inhibitor of FoxM1 in mouse hepatocellular carcinoma treatment. J Clin Invest 2007; 117: 99-111.

\title{
CARMA1 is a novel regulator of T-ALL disease and leukemic cell migration to the CNS
}

Leukemia (2017) 31, 255-258; doi:10.1038/leu.2016.272

Pediatric acute lymphoblastic leukemia (ALL) is the most common type of malignancy in children with $15 \%$ bearing markers for the T-lineage (T-ALL) and $20 \%$ of T-ALL patients still suffering disease relapse. Many genes are implicated in T-ALL disease, including Notch, and Notch mutations have been identified in a majority of T-ALL patients. ${ }^{1}$ Patients with T-ALL relapse with disease usually in the central nervous system (CNS). ${ }^{2}$ Despite the use of intrathecal therapy and cranial radiation, successful management of CNS disease continues to be problematic in T-ALL. ${ }^{3}$ Few molecules are known to be associated with T-ALL CNS disease including CCR7, a chemokine receptor normally expressed on naive T cells. ${ }^{4}$ CXCR4 has also recently been shown to have a role in T-ALL initiation and progression. ${ }^{5-7}$ The involvement of chemokine receptors as well as other molecules regulating cell motility suggests that regulation of T-ALL cell migration is likely to have an important role in T-ALL.

Cell signaling molecules that drive normal T-cell proliferation, such as PKC $\theta$, can regulate T-ALL. ${ }^{8}$ PKC $\theta$ activates CARMA1 (also known as CARD11) bringing together Bcl10 and MALT1 leading to NF-KB activation. ${ }^{9}$ Although higher expression of CARMA1 has been seen in a small subset of T-ALL patients, little is known about how CARMA1 might regulate T-ALL. ${ }^{10}$ To study the potential role of CARMA1 in T-ALL disease, we used shRNA to knock down CARMA1 expression in the human CEM T-ALL cell line expressing luciferase and GFP (called CARMA1KD), with the level of knockdown $\sim 50 \%$ (Supplementary Figure 1A). We transferred the CARMA1KD CEM cells into the immunodeficient NOD.Cg-Prkdc scid $\| 2 \mathrm{rg}^{\mathrm{tm} 1 \mathrm{Wjl}} / \mathrm{SzJ}$ IL2RG (NSG) mice. Animals receiving CARMA1KD T-ALL cells survived significantly longer than animals receiving parental CEM cells (Figure 1a). We also used a Notch1- $\Delta$ E-induced mouse T-ALL model in which hematopoietic precursors from WT C57BI/6 (called WT/WT Notch1 $\Delta \mathrm{E}$; labeled WT) or CARMA1 - /(WT/CARMA1 - / - Notch1 $\triangle \mathrm{E}$; labeled CARMA1KO) animals were transduced with Notch1 $\triangle \mathrm{E}-\mathrm{GFP}$ and develop T-ALL. Like the xenograft model, we found that WT/CARMA1-/Notch1 $1 \mathrm{E}$ (CARMA1KO) animals survived significantly longer than WT/WT Notch1 $\triangle \mathrm{E}$ (WT; Figure 1b).

We then asked whether CARMA1 is also involved in human T-ALL disease. Using microarray analyses from patient T-ALL cells, we compared CARMA1 expression in bone marrow (BM) cells from T-ALL patients with BM cells from a non-leukemia control population. CARMA1 expression was significantly higher in the T-ALL patients cells compared with non-leukemic cells (Figure 1c) and other tumor types including lung, liver and breast (Figure 1d).
These results, along with the animal models, demonstrate that CARMA1 is an important regulator of T-ALL disease.

We assessed proliferation of CARMA1KD cells compared with parental CEM T-ALL cells. Surprisingly, we found that CARMA1KD showed no difference in basal in vitro proliferation measured by cell growth in culture (Supplementary Figure 1B) or baseline T-ALL cell apoptosis measured by Annexin $V$ staining (Supplementary Figure 1C) compared with parental CEM T-ALL cells. We also assessed tumor growth using in vivo bioluminescent luciferase imaging of CEM cells. Animals that received parental CEM cells showed a higher T-ALL tumor load than animals receiving CARMA1KD CEM cells at weeks 1,2 and 3 (Supplementary Figures $2 \mathrm{~A}$ and B). We examined T-ALL cell accumulation in specific tissues using anti-human CD45 and GFP expression to identify CEM cells by flow cytometry. CARMA1KD CEM cells were significantly decreased in the liver, but we saw no difference in the $\mathrm{BM}$ or in spleens between parental and CARMA1KD CEMs (Figure 1e). Using the NOTCH-induced T-ALL model, we found that CARMA1-/- T-ALL cells accumulated less in lymph node, but not in BM, spleen or liver (Supplementary Figure 2C).

Our previous results suggested CARMA1 may regulate differential migration patterns to specific organs. We used the transwell migration assay to compare the migration of parental and CARMA1KD CEM cells in vitro. We assessed T-ALL cell migration toward CCL21, the ligand for CCR7 and found that CARMA1KD CEM cells showed a significant decrease in migration to CCL21 and LFA-1 ligand ICAM1 compared with parental CEM cells (Figure 2a). CCR7 has been shown to regulate T-ALL migration specifically to the CNS. ${ }^{4}$ Using the CEM xenograft model, we could detect the presence of parental CEM cells in both the calvarial BM (indicated by ${ }^{*}$ ) as well as the meninges of the CNS (arrows, Figure 2b). ${ }^{11}$ In contrast, animals receiving CARMA1KD CEM cells showed little to no accumulation in the meninges, but still showed accumulation in the BM. We measured the total area of meningeal T-ALL infiltrate in four coronal sections per mouse and parental CEM cells showed higher levels of cell infiltration compared with CARMA1KD CEM cells (Supplementary Table 1A). We also analyzed CNS infiltration of T-ALL cells in the NOTCH model of T-ALL and found similar results to the xenograft model (Supplementary Figure 3, Supplementary Table 1B). These data show that CARMA1 expression in T-ALL cells leads to an increase in T-ALL cell infiltration into the CNS.

We then evaluated CARMA1 expression in a cohort of T-ALL patients who initially presented with T-ALL cells in the CNS versus 


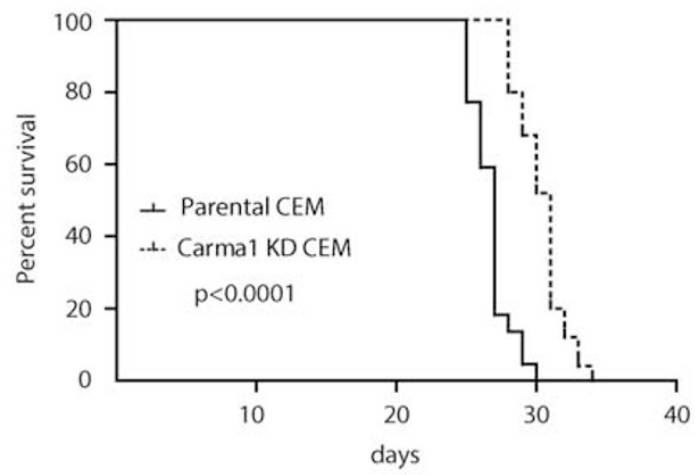

C

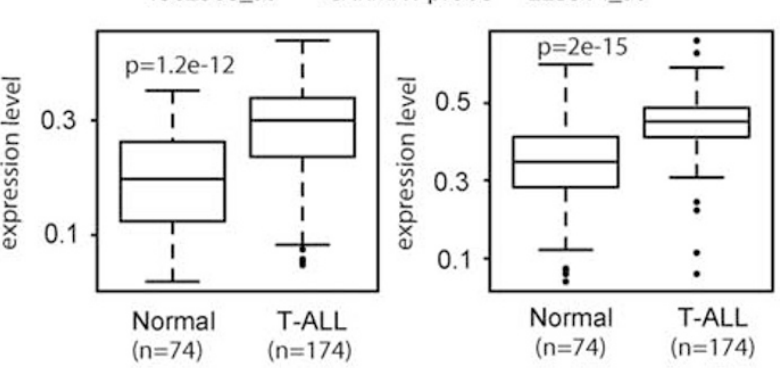

b

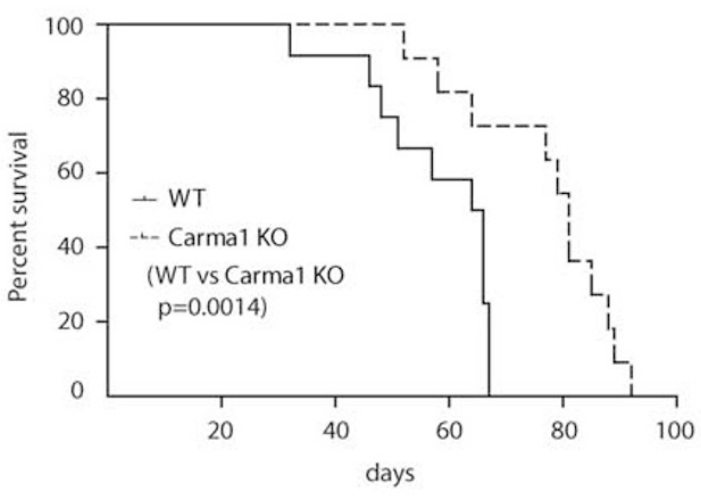

d

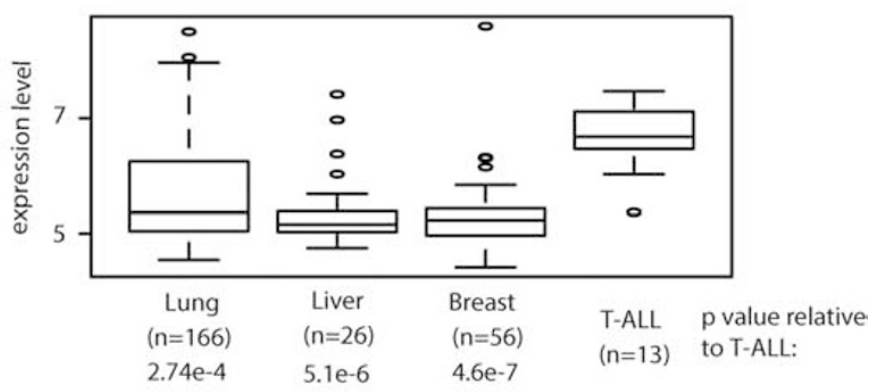

e

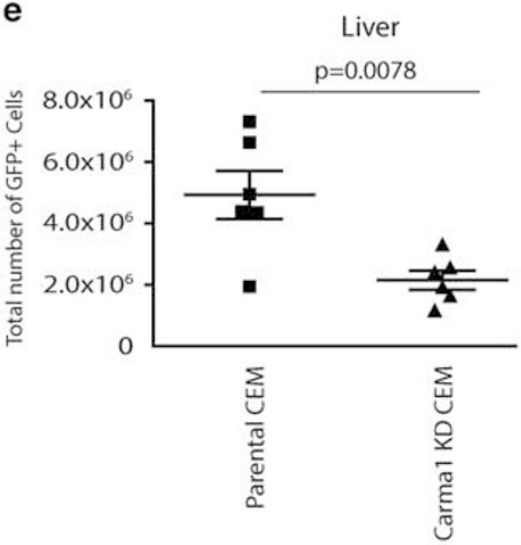

BM

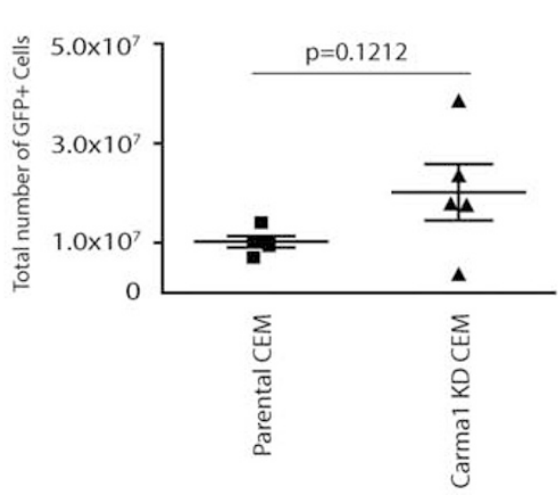

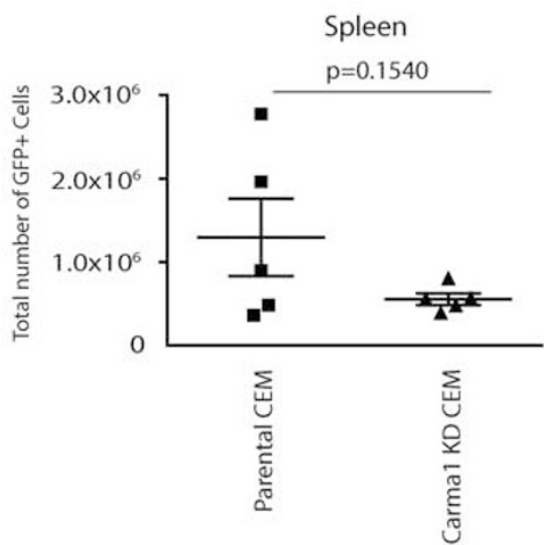

Figure 1. CARMA1 expression exacerbates T-ALL disease. (a) Survival curves of NSG recipients xenografted with parental $(n=22)$ or CARMA1KD $(n=25)$ CEM cells. (b) Lin ${ }^{-}$cells from bone marrow of C57BI/6 and CARMA1 - / - mice were transfected with Notch1 $\Delta \mathrm{E}-\mathrm{GFP}$ and transferred into irradiated B6.LY5.1 animals. Survival curves for WT/WT Notch1 $\Delta \mathrm{E}$ (labeled WT) animals $(n=12)$ and WT/CARMA1 - / - Notch1 $\Delta \mathrm{E}$ animals (labeled CARMA1KO; $n=11)$ are shown. For $(\mathbf{a})$ and $(\mathbf{b}), P$-values were determined using the Mantel-Cox test. (c) Data from nonleukemic bone marrow and T-ALL patient bone marrow from GEO accession \# GSE13204 were analyzed using the indicated CARMA1 probes. (d) Data from CCLE were analyzed for CARMA1 as shown for indicated solid tumor cell lines and T-ALL. For comparisons in (c) and (d), the number of patients in each group is shown by $n$ and $P$-values calculated using the Mann-Whitney Wilcoxon test and exact $P$-values shown. (e) Specified organs were processed from animals receiving parental or CARMA1KD cells at week 3 and GFP+ CEM cells were enumerated. $P$-values shown used the Student's $t$-test.

patients who showed no CNS disease at time of diagnosis. T-ALL patients with CNS involvement showed significantly higher expression of both CARMA1 probes in the microarray compared with patients with no CNS involvement (Figure 2c). These results suggest that CARMA1 may specifically regulate T-ALL cell migration to the CNS.

In this study, we show that CARMA1 is a novel regulator of T-ALL disease in animal models as well as data from T-ALL patients. CARMA1 expression is associated with increased T-ALL disease severity as well as leukemia cell accumulation in the CNS. Many studies, including whole-genome studies, have identified novel regulators of T-ALL disease. ${ }^{12}$ A previous study showed that
CARMA1 is upregulated in a small group of T-ALL patients ${ }^{10}$ but did not find a specific role for CARMA1 in CNS disease. Only a few molecules including chemokine receptors CCR7 and CXCR4 have been specifically shown to be involved in CNS disease in T-ALL., ${ }^{4}$ We find that animals with CARMA1-/- T-ALL show similar survival advantage to CCR7 - / - T-ALL (Supplementary Figure 4). We also find that CARMA1 colocalizes with CCR7 (Supplementary Figure 5) and CARMA1KD cells show decreased migration to the CCR7 ligand CCL21 in vitro. Although some of our results suggest that CARMA1 affects migration of T-ALL cells directly downstream of CCR7, it is also possible that CARMA1 and CCR7 independently regulate T-ALL cell migration. In T cells, CXCR4 has also been 
a

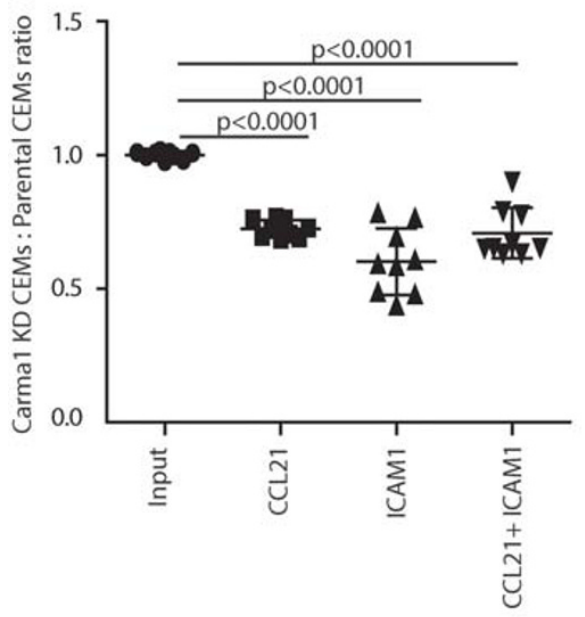

b
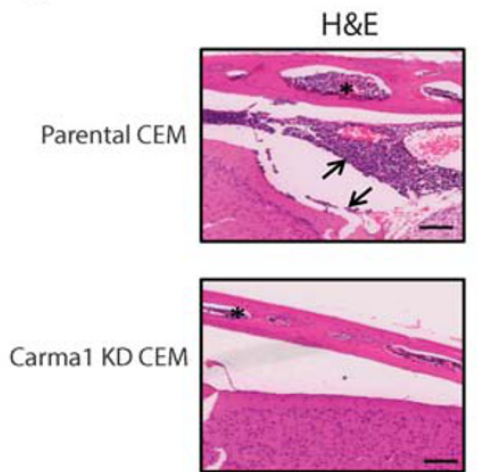

a-human CD45
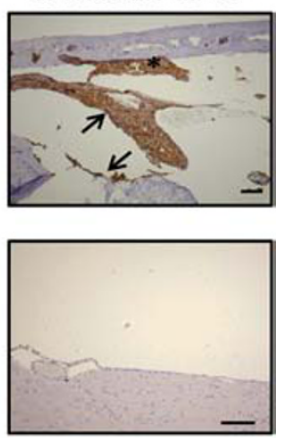

C
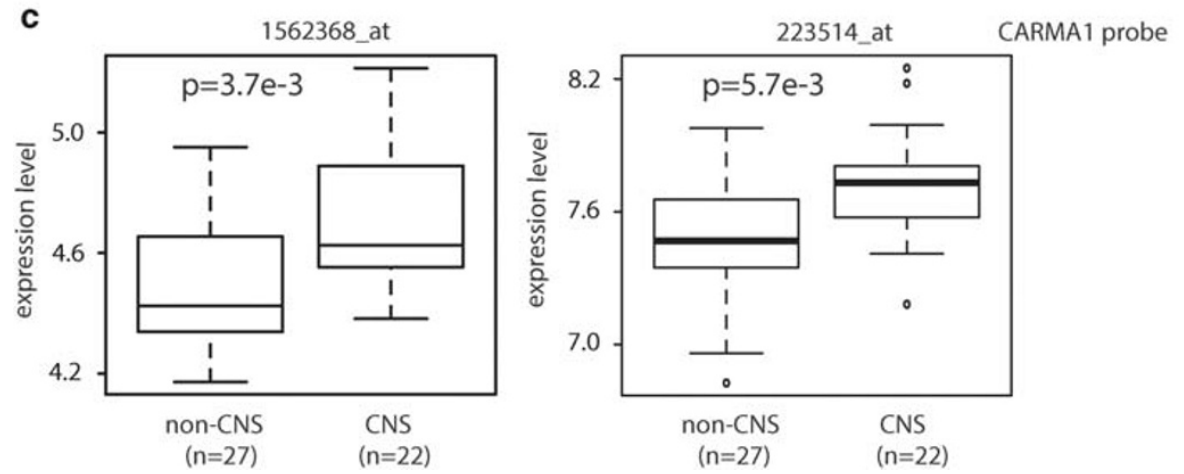

Figure 2. CARMA1 regulates T-ALL cell migration to the CNS. (a) Parental and CARMA1KD CEM cells were assayed for migration by transwell assay toward CCL21, ICAM1 or CCL21+ICAM1. P-values shown were calculated with the Student's $t$-test. (b) Heads of xenotransplanted animals with parental or CARMA1KD CEM cells and processed for H\&E (in Methods). Images of sections through skull and meninges of two representative mice (of four) and stained for H\&E (left hand panel) or anti-human CD45 (right hand panel). Meningeal infiltration indicated by thick arrow and bone marrow by *. Scale bar represents $100 \mu \mathrm{m}$. (c) Data from study 9404 GEO accession \# GSE14615 were analyzed for indicated CARMA1 probes. For all comparisons, the number of patients in each group is shown by $n$ and $P$-values calculated using the MannWhitney Wilcoxon test and exact $P$-values shown.

colocalized with ZAP70, suggesting that cross talk between chemokine receptors and intracellular signaling pathways such as CCR7 and CARMA1 may act both in normal and leukemic cells to regulate cell migration. ${ }^{13}$

In normal T cells, TCR activation activates PKC $\theta$ phosphorylation of CARMA1, leading to NF-kB activation. ${ }^{9}$ We previously showed that PKC $\theta$ and CARMA1 regulate naive T-cell migration ${ }^{14,15}$ and PKC $\theta$ has been shown to have a role in T-ALL. ${ }^{8}$ We find that animals receiving PKCOKD CEM cells show similar survival to those receiving parental CEM cells (Supplementary Figures $6 \mathrm{~A}$ and $\mathrm{B}$ ). This suggests that unlike naive $T$ cells, in which PKC $\theta$ activates CARMA1, T-ALL cells may activate CARMA1 independently of PKCO. CARMA1 may be aberrantly upregulated in T-ALL, leading to disease progression and migration of leukemic cells to the CNS. In the future, inhibitors of CARMA1 or pathways that lead to CARMA1 activation may prove therapeutically useful in controlling CNS disease in T-ALL.

\section{CONFLICT OF INTEREST}

The authors declare no conflict of interest.

\section{ACKNOWLEDGEMENTS}

This work was supported by NIH 1R01AI097202 (JLC), the Spatiotemporal Modeling Center (P50 GM085273), UNM Cancer Center (P30-CA118100, SRO), UNM HSC IRG-92-024-16 (JLC)
NIH RL5MD009592 (DJT), Kay Kendall Leukaemia Fund (KKL454) (CH) and the Chief Scientists' Office (SCD/08, CH). We thank Richard Harvey, Will Kanagy and Paulus Mrass of UNM; Clare Orange and Lynn Stevenson, University of Glasgow and Think Pink, Scotland; Ben Alderete, Brian Hall and Tim Chang at EMD Millipore.

SR Oruganti ${ }^{1}$, DJ Torres ${ }^{2}$, S Krebsbach $^{1}$, F Asperti-Boursin ${ }^{1}$, J Winters ${ }^{1}$, K Matlawska-Wasowska $^{3}$, SS Winter ${ }^{3}, \mathrm{CH}$ Halsey $^{4}$ and JL Cannon ${ }^{1,5}$

${ }^{1}$ Department of Molecular Genetics and Microbiology, University of New Mexico Health Sciences Center, Albuquerque, Mexico;

${ }^{2}$ Department of Mathematics and Physical Science, Northern New Mexico College, Espanola, Mexico;

${ }^{3}$ Department of Pediatrics, University of New Mexico Health Sciences Center, Albuquerque, Mexico;

${ }^{4}$ Wolfson Wohl Cancer Research Centre, Institute of Cancer Sciences, College of Medical, Veterinary and Life Sciences, University of Glasgow and Royal Hospital for Children, Glasgow, Scotland and ${ }^{5}$ Department of Pathology, University of New Mexico Health Sciences Center, Albuquerque, Mexico E-mail: jucannon@salud.unm.edu

\section{REFERENCES}

1 Weng AP, Ferrando AA, Lee W, JPt Morris, Silverman LB, Sanchez-Irizarry C et al. Activating mutations of NOTCH1 in human T cell acute lymphoblastic leukemia. Science 2004; 306: 269-271. 
2 Pui $\mathrm{CH}$, Thiel E. Central nervous system disease in hematologic malignancies: historical perspective and practical applications. Semin Oncol 2009; 36(4 Suppl 2): S2-S16.

3 Asselin BL, Devidas M, Wang C, Pullen J, Borowitz MJ, Hutchison R et al. Effectiveness of high-dose methotrexate in T-cell lymphoblastic leukemia and advanced-stage lymphoblastic lymphoma: a randomized study by the Children's Oncology Group (POG 9404). Blood 2011; 118: 874-883.

4 Buonamici S, Trimarchi T, Ruocco MG, Reavie L, Cathelin S, Mar BG et al. CCR7 signalling as an essential regulator of CNS infiltration in T-cell leukaemia. Nature 2009; 459: 1000-1004.

5 Gomez AM, Martinez C, Gonzalez M, Luque A, Melen GJ, Martinez J et al. Chemokines and relapses in childhood acute lymphoblastic leukemia: a role in migration and in resistance to antileukemic drugs. Blood Cells Mol Dis 2015; 55: 220-227.

6 Passaro D, Irigoyen M, Catherinet C, Gachet S, Da Costa De Jesus C, Lasgi C et al. CXCR4 is required for leukemia-initiating cell activity in T cell acute lymphoblastic leukemia. Cancer Cell 2015; 27: 769-779.

7 de Lourdes Perim A, Amarante MK, Guembarovski RL, de Oliveira CE, Watanabe MA. CXCL12/CXCR4 axis in the pathogenesis of acute lymphoblastic leukemia (ALL): a possible therapeutic target. Cell Mol Life Sci 2015; 72: $1715-1723$.

8 Felli MP, Vacca A, Calce A, Bellavia D, Campese AF, Grillo R et al. PKC theta mediates pre-TCR signaling and contributes to Notch3-induced T-cell leukemia. Oncogene 2005; 24: 992-1000.

9 Thome M, Charton JE, Pelzer C, Hailfinger S. Antigen receptor signaling to NF-kappaB via CARMA1, BCL10, and MALT1. Cold Spring Harb Perspect Biol 2010; 2: $\mathrm{a} 003004$.
10 Ma Y, Liao Z, Xu Y, Zhong Z, Wang X, Zhang F et al. Characteristics of CARMA1BCL10-MALT1-A20-NF-kappaB expression in T cell-acute lymphocytic leukemia. Eur J Med Res 2014; 19: 62.

11 Williams MT, Yousafzai $\mathrm{Y}$, Cox C, Blair A, Carmody R, Sai $\mathrm{S}$ et al. Interleukin-15 enhances cellular proliferation and upregulates CNS homing molecules in pre-B acute lymphoblastic leukemia. Blood 2014; 123: 3116-3127.

12 Van Vlierberghe P, Ferrando A. The molecular basis of T cell acute lymphoblastic leukemia. J Clin Invest 2012; 122: 3398-3406.

13 Kumar A, Humphreys TD, Kremer KN, Bramati PS, Bradfield L, Edgar CE et al. CXCR4 physically associates with the T cell receptor to signal in T cells. Immunity 2006; 25: 213-224.

14 Cannon JL, Asperti-Boursin F, Letendre KA, Brown IK, Korzekwa KE, Blaine KM et al. PKCtheta regulates $T$ cell motility via ezrin-radixin-moesin localization to the uropod. PLoS One 2013; 8: e78940.

15 Letendre K, Donnadieu E, Moses ME, Cannon JL. Bringing statistics up to speed with data in analysis of lymphocyte motility. PLoS One 2015; 10: e0126333.

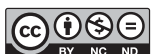

This work is licensed under a Creative Commons AttributionNonCommercial-NoDerivs 4.0 International License. The images or other third party material in this article are included in the article's Creative Commons license, unless indicated otherwise in the credit line; if the material is not included under the Creative Commons license, users will need to obtain permission from the license holder to reproduce the material. To view a copy of this license, visit http:// creativecommons.org/licenses/by-nc-nd/4.0/

(c) The Author(s) 2017

Supplementary Information accompanies this paper on the Leukemia website (http://www.nature.com/leu)

\section{Expansion of $\mathrm{Th}_{1}$-like $\mathrm{V} \gamma_{9} \mathrm{~V} \delta_{2} \mathrm{~T}$ cells by new-generation IMiDs, lenalidomide and pomalidomide, in combination with zoledronic acid}

\section{Leukemia (2017) 31, 258-262; doi:10.1038/leu.2016.273}

Multiple myeloma (MM) still remains incurable even with the implementation of novel therapeutic modalities, leading to the idea to develop various forms of immunotherapies. One such approach is the application of $\gamma \delta$ T cells. Similar to NK cells, $\gamma \delta$ $\mathrm{T}$ cells are important effectors in the first-line defense against infections and tumors, and have a critical role in host defense and tumor surveillance. ${ }^{1-3}$ In human, $\gamma \delta \mathrm{T}$ cells are divided into two predominant subpopulations by T-cell receptor $\mathrm{V}$ gene usage, namely $V \delta_{1} \gamma \delta T$ cells and $V \gamma_{9} V \delta_{2} \gamma \delta T\left(\gamma \delta_{2} T\right)$ cells. $\gamma \delta_{2} T$ cells

Figure 1. $\gamma \delta_{2}$ T-cell expansion and activation by Zol in combination with new-generation IMiDs. (a, b) $\gamma \delta_{2}$ T-cell expansion by LEN and Zol in combination. PBMCs isolated from a normal donor (\# 1) were cultured for 1 week alone or in the presence of Zol at $1 \mu \mathrm{m}$ with or without LEN at $1 \mu \mathrm{m}$ or IL-2 at $100 \mathrm{IU} / \mathrm{ml}$ as indicated (a). Then, the cells were analyzed by flow cytometry to determine a distribution of $\gamma \delta_{2} T$ cells. V $\delta_{2}$-positive and CD3-positive cells accumulated in the right upper columns represent $\gamma \delta_{2}$ T cells. PBMCs isolated from additional six normal donors (\# 2-7) (left) or $6 \mathrm{MM}$ patients (right) were cultured for 1 week alone or with Zol at $1 \mu \mathrm{m}$ in combination with either LEN at $1 \mu \mathrm{M}$ or IL-2 at 100 IU/ml, followed by flow cytometric analysis to determine the expansion of $\gamma \delta_{2} T$ cells $(\mathbf{b})$. Results are expressed as a \% distribution of $\gamma \delta_{2} T$ cells within CD3-positive cells. (c) Induction of Th were stained by PE-labeled anti- $\mathrm{V}_{2}$ monoclonal antibody together with FITC-labeled monoclonal antibodies for the indicated molecules. Intracellular levels of IFN- $\gamma$ and Foxp3 in $\gamma \delta_{2}$ T cells are indicated in red lines (upper). Surface levels of NKG2D and DNAM-1 and intracellular levels of perforin and granzyme B in $\gamma \delta_{2} T$ cells are shown in red lines (lower). Baseline expression of these molecules in $\gamma \delta_{2} T$ cells without treatment with LEN plus Zol was indicated by blue lines. Gray areas indicate background staining with lgG isotype controls. (d) The cytotoxic activity against MM cells of $\gamma \delta_{2}$ T cells expanded with LEN and Zol in combination. PBMCs from a normal donor were cultured for 1 week with LEN and Zol in combination. After expansion, whole PBMCs were used as effectors and added at the indicated effector to target (E:T) ratios and co-cultured in triplicate with PKH-labeled MM cell lines ( $\square$, RPMI8226; $\square$, KMS-11; $\square$, U266) (upper), and PKH-labeled primary MM cells (lower). The cells were then stained with 7-amino-actinomycin D (7-AAD) to detect dead cells within the PKH26-lebeled MM cells by flow cytometry. Results are expressed as a \% distribution of dead cells within MM cells. (e) $\gamma \delta_{2}$ T-cell expansion by Zol in combination with LEN or POM. PBMCs isolated from normal donors (\# 8-17) were cultured for 1 week alone or upon treatment as indicated, followed by flow cytometric analysis to determine the expansion of $\mathrm{V}_{2} \gamma \delta \mathrm{T}$ cells. Zol, LEN, POM and IL-2 were added at 1, 1, $0.1 \mu \mathrm{M}$ and $100 \mathrm{IU} / \mathrm{ml}$, respectively. Results are expressed as a \% distribution of $\gamma \delta_{2}$ T cells in CD3-positive cells. 